\title{
Supplementary Text:
}

\section{Sensitivity analysis with medication and clinical chemistry}

We used sensitivity analysis to assess the effect of additional covariates on T2D metabolite associations. For sensitivity analysis, in addition to the covariates mentioned in the Materials and Methods, we added each of the following covariates separately and investigated their impact on the final result: HDL, LDL, TRI, creatinine, blood urea nitrogen (BUN), and medication. Medication consisted of statins; antihypertensives using a combination variable of use of angiotensin-converting enzyme [ACE], angiotensin II receptor blockers [ARBs], and calcium channel blockers [CC], referred to as ACE_ARB_CC; beta-blockers; thyroxine; and aspirin. Supplementary Table 3 shows the results of sensitivity analysis, with metabolites that have been affected after adjusting for those covariates. After adjusting for statins, 18 metabolites associated with T2D became nonsignificant. Adjustment for ACE_ARB_CC, aspirin, BUN, and creatinine affected 18, 10, eight, and three metabolites, respectively. Statins were associated with the xenobiotics 2-hydroxyhippurate (salicylurate) and salicylate, along with seven other metabolites. Aspirin was associated with three xenobiotics - O-sulfo-L-tyrosine, 2-hydroxyhippurate (salicylurate), and salicylate and two unknown metabolites. After creatinine and BUN adjustment, three and eight metabolites became non-significant, respectively, including O-sulfo-L-tyrosine and metabolites from leucine/isoleucine/valine metabolism, among others. Adjustment for TRI, HDL, and LDL revealed 34, 21, and 20 affected metabolites, respectively.

\section{Shared and Unique metabolites:}

We visualized significant metabolites of super-pathways in the eight clinical variables while highlighting the top most significant hits in either T2D or TRI (Figure 2a). We then identified 17 sub-pathways ( $\geq 6$ metabolites) in amino acids ( 6 sub-pathways), lipids (10 sub-pathways) and peptides (one sub-pathway) to identify significant pathway associations shared between clinical variables (heatmap in Figure $\mathbf{2 b}$ ). Based on the shared metabolic perturbations among the clinical variables, we computed correlations between the studied phenotypes (T2D, BMI, retinopathy, LDL, HDL, TRI, and LDL /HDL ratio) using regression statistics (sign[beta) ${ }^{*}-\log 10[p$-value]) with the 373 metabolites (Supplementary Figure 3). Boxplots of selected metabolites associated with five or more variables are shown in Supplementary Figure 4a-b, and those associated only with T2D, retinopathy, or TRI are shown in Supplementary Figures 4c-h.

\section{GGMs:}

The second largest subnetwork consisted of sphingomyelins (20 metabolites, Supplementary Figure 5b), which were associated with two or more phenotypes, followed by two fatty acid subnetworks with 17 and 13 metabolites, respectively (Figures 3a,3e). The first fatty acid subnetwork comprised PUFAs, phospholipids, and monoacylglycerols and had seven metabolites associated only with T2D and seven associated only with TRI. The second subnetwork included eight metabolites associated with TRI, mainly long-chain fatty acids and PUFAs. Subnetworks which contained metabolites associated only with T2D are: dipeptides and unknowns (Figure 3f), monohydroxy fatty acid metabolism (Figure 3b), carbohydrates and sugars (Figure 3c), and a fungal/xenobiotic/unknowns subnetwork (Figure 3d). Finally, 
BCAAs/phenylalanine and tyrosine subnetworks (Supplementary Figures $\mathbf{5 c , e}$ ) and a glucoronides/unknowns subnetwork were identified (Supplementary Figure 5d).

\section{Extended Discussion:}

Among the most shared pathways (i.e. shared between four or more clinical outcomes) were the associations of T2D, BMI, LDL, retinopathy, and LDL/HDL with sphingolipids, and of T2D, BMI, LDL, and TRI with phospholipids, lysolipids, and fatty acids (monohydroxy, branched, acylcarnitine). Additionally, T2D, $\mathrm{BMI}$, and TRI shared associations with BCAAs and carbohydrates (glycolysis, glycogen, fructose, mannose, pentose, amino sugar, and advanced glycation end-products [AGEs]), whereas T2D, BMI, and retinopathy shared associations with amino acids of the phenylalanine and tyrosine pathway, 12 purine/pyrimidines, and lysolipids. T2D, TRI, and retinopathy shared associations with 11 peptides.

In addition to those mentioned above, T2D and TRI also shared associations with xenobiotics, LCFAs, and PUFAs. Finally, T2D shared only a few associations (methionine, cysteine, SAM, and taurine pathways) with TRI, LDL/HDL, and retinopathy, and the urea cycle with BMI, whereas T2D was predominantly associated with those pathways. Of all clinical variables, TRI showed the strongest associations with metabolites from the phospholipids, diacylglycerol, and monoacylglycerol pathways, among others.

\section{Sphingomyelins:}

Our cohort uncovered several sphingomyelins with distinct chemical structures, correlated as shown in the GGM subnetwork (Figure 3 and Supplementary Figure 5). Sphingosine, the end product of sphingomyelin degradation [1], was elevated in our cohort, with a corresponding decrease in sphingomyelin levels in T2D. Sphingomyelins were previously associated with insulin resistance in clinical studies [2] and with diabetic nephropathy pathogenesis [3], and sphingomyelin levels were used to discriminate among healthy, T2D, and diabetic nephropathy patients [4]. Sphingomyelins are also involved in the pathogenesis of diabetic retinopathy [3], neuropathy [5] and cardiovascular disease [6].

\section{Lysolipids, Phospholipids \& Plasmalogens:}

The anti-inflammatory and glucose-lowering effects of lysolipids make them good indicators of metabolic disorder, obesity, and cardiovascular disease risk for T2D patients [7]. Lysolipids were decreased with T2D in our cohort but increased with high TRI levels. However, phospholipids that significantly increased with TRI also increased with T2D. Altered phospholipid metabolism is central to the pathogenesis of metabolic diseases [2] and is associated with insulin resistance and sensitivity [8]. We found that 17 ethanolamineand choline-containing phospholipid metabolites-the most abundant phospholipids in human metabolism [9] - were altered in T2D (5 increased and 12 decreased).

Consistent with a decrease in PUFAs, plasmalogen and lysoplasmalogen, which are enriched in PUFA (arachidonate), were decreased in T2D. Plasmalogens, the primary constituents of phospholipids [10], are inversely associated with T2D and cardiovascular disease [11] [12]. They may be used as an oxidative stress marker for diabetic patients [13], as they are thought to act as endogenous antioxidants; thus, decreased concentrations indicate higher oxidative stress [14]. 


\section{Oxidative Stress:}

Excess free fatty acids (FFA) can lead to insulin resistance and elevated tissue lipolysis. This then leads to additional elevation of plasma FFA, as observed in obese individuals, and increases biosynthesis of signaling molecules such as DAG and sphingolipids. This can also be explained by the production of

adipokines, which often results in the release of fatty acids from adipose tissue and their deposition in distal tissues; this promotes the development of lipotoxicity. The resulting oxidative stress has been shown to trigger ceramide accumulation. Moreover, elevated sphingolipid synthesis contributes to cell death through endoplasmic reticulum stress, which has been shown to contribute to the insulin resistance that leads to the development of T2D. Excess fatty acids have been found to mediate lipotoxic cellular dysfunction through such mitochondrial dysfunction, and mitochondrial overload with substrates for $\beta$ oxidation can increase cell oxidative stress.

\section{Amino Acids and Oxidative Stress:}

3-HIB is known to be secreted from muscle cells and to activate endothelial fatty acid transport. It also stimulates muscle fatty acid uptake in vivo; promotes lipid accumulation in muscle, leading to insulin resistance in mice; regulates the trans-endothelial flux of fatty acids; and links the regulation of fatty acid flux to BCAA catabolism [15].

Glutathione, which is a major intracellular antioxidant and plays a key role in reducing the effects of oxidative stress, was found to be decreased in T2D patients in our cohort (oxidized cys-gly, cysteineglutathione disulfide) compared to controls, in agreement with what was previously found in diabetic patients with known microvascular complications [16].

Taurine is another important amino acid biomarker for T2D because of its antioxidant effect, known to be related to obesity and non-alcoholic fatty liver disease (NAFLD) [17]. All metabolites involved in the urea cycle were decreased, and amino acids involved in the urea cycle are known to be associated with inflammatory markers and oxidative stress. Arginine, threonine, and metabolites in the alanine and aspartate metabolism pathway are associated chronic heart disease in diabetic patients [18].

\section{T2D metabolites and link to oxidative stress}

Metabolites associated only with T2D are involved in kidney function or chronic kidney disease. These include 1,5-anhydroglucitol, creatinine and guanidinoacetate (creatine metabolism) [19], Nacteylglutamine and pyroglutamine (glutamate metabolism), pseudouridine, tartronate (novel to T2D) and 4-hydroxychlorothalonil [20] [21]. Uric acid and creatinine are positively correlated with $\beta$-cell function in T2D patients [19]. BCAA metabolites associated only with T2D include 3-methylglutaconate (novel metabolite) of the leucine pathway [22] and isobutyrylglycine and isovalerylglycine, which are both related to insulin resistance [23]. Proline is known to protect mammalian cells against oxidative stress [24], and the novel associations of thioproline and N-methylproline are associated only with T2D; thioproline, which is an antioxidant that protects the body from oxidative stress [25], was decreased. Also novel and associated only with T2D were $\mathrm{N}$-acetylarginine, which affects oxidative stress in rats [26], and dopamine sulfate. Dopamine is of special importance in neuronal redox-homeostasis and viability and is 
linked to oxidative stress; its degradation results in the production of ROS, and its oxidation can lead to endogenous neurotoxins, while some dopamine derivatives also show antioxidative effects [27].

\section{Metabolic Signatures:}

Figure 4e shows a signature that combines an LCFA (palmitate), phenylalanine and a BCAA (3-methyl-2oxovalerate) among other lipids that are at their lowest levels in the largest portion of retinopathy patients having the lowest levels of TRI. Palmitate, a biomarker for T2D [28], is increased in T2D compared to controls, yet shows the highest levels in the patients with the highest TRI levels. It has been reported to induce toxicity via oxidative stress, leading to reactive oxygen species (ROS) generation and $\mathrm{Ca}^{2+}$-mediated pathogenic changes [28]. It is also involved in the de novo biosynthesis of sphingolipids [29] and is known to be perturbed in T2D complications such as retinopathy and nephropathy; its accumulation in T2D patients with persistent hyperglycemia accelerates retinal mitochondrial DNA damage via glucolipotoxicity, and it is involved in the progression of diabetic nephropathy [30]. The low levels of phenylalanine and 3-methyl-2-oxovalerate in the highest portion of retinopathy patients requires investigation of the importance of administering those amino acids in retinopathy patients.

[1] C. Kunzelmann-Marche, J.M. Freyssinet, and M.C. Martinez, Loss of plasma membrane phospholipid asymmetry requires raft integrity. Role of transient receptor potential channels and ERK pathway. J Biol Chem 277 (2002) 19876-81.

[2] W. Chang, G.M. Hatch, Y. Wang, F. Yu, and M. Wang, The relationship between phospholipids and insulin resistance: From clinical to experimental studies. Journal of Cellular and Molecular Medicine 23 (2019) 702-710.

[3] D. Zhu, P.G. Sreekumar, D.R. Hinton, and R. Kannan, Expression and regulation of enzymes in the ceramide metabolic pathway in human retinal pigment epithelial cells and their relevance to retinal degeneration. Vision Res 50 (2010) 643-651.

[4] C. Zhu, Q.-I. Liang, P. Hu, Y.-m. Wang, and G.-a. Luo, Phospholipidomic identification of potential plasma biomarkers associated with type 2 diabetes mellitus and diabetic nephropathy. Talanta 85 (2011) 1711-1720.

[5] J. Wang, L. Badeanlou, J. Bielawski, T.P. Ciaraldi, and F. Samad, Sphingosine kinase 1 regulates adipose proinflammatory responses and insulin resistance. Am J Physiol Endocrinol Metab 306 (2014) E756-E768.

[6] Z.H. Alshehry, P.A. Mundra, C.K. Barlow, N.A. Mellett, G. Wong, M.J. McConville, J. Simes, A.M. Tonkin, D.R. Sullivan, E.H. Barnes, P.J. Nestel, B.A. Kingwell, M. Marre, B. Neal, N.R. Poulter, A. Rodgers, B. Williams, S. Zoungas, G.S. Hillis, J. Chalmers, M. Woodward, and P.J. Meikle, Plasma Lipidomic Profiles Improve on Traditional Risk Factors for the Prediction of Cardiovascular Events in Type 2 Diabetes Mellitus. Circulation 134 (2016) 1637-1650.

[7] B. García-Fontana, S. Morales-Santana, C. Díaz Navarro, P. Rozas-Moreno, O. Genilloud, F. Vicente Pérez, J. Pérez del Palacio, and M. Muñoz-Torres, Metabolomic profile related to cardiovascular disease in patients with type 2 diabetes mellitus: A pilot study. Talanta 148 (2016) 135-143.

[8] E. Candi, M. Tesauro, C. Cardillo, A.M. Lena, F. Schinzari, G. Rodia, G. Sica, P. Gentileschi, V. Rovella, M. Annicchiarico-Petruzzelli, N. Di Daniele, and G. Melino, Metabolic profiling of visceral adipose 
tissue from obese subjects with or without metabolic syndrome. Biochemical Journal 475 (2018) 1019-1035.

[9] M. Borkman, L.H. Storlien, D.A. Pan, A.B. Jenkins, D.J. Chisholm, and L.V. Campbell, The relation between insulin sensitivity and the fatty-acid composition of skeletal-muscle phospholipids. $\mathrm{N}$ Engl J Med 328 (1993) 238-44.

[10] M.C.F. Messias, G.C. Mecatti, D.G. Priolli, and P. de Oliveira Carvalho, Plasmalogen lipids: functional mechanism and their involvement in gastrointestinal cancer. Lipids in Health and Disease 17 (2018) 41.

[11] P.J. Meikle, G. Wong, C.K. Barlow, J.M. Weir, M.A. Greeve, G.L. Maclntosh, L. Almasy, A.G. Comuzzie, M.C. Mahaney, A. Kowalczyk, I. Haviv, N. Grantham, D.J. Magliano, J.B.M. Jowett, P. Zimmet, J.E. Curran, J. Blangero, and J. Shaw, Plasma Lipid Profiling Shows Similar Associations with Prediabetes and Type 2 Diabetes. PLOS ONE 8 (2013) e74341.

[12] B. Stratmann, K. Richter, R. Wang, Z. Yu, T. Xu, C. Prehn, J. Adamski, T. Illig, D. Tschoepe, and R. Wang-Sattler, Metabolomic Signature of Coronary Artery Disease in Type 2 Diabetes Mellitus. International Journal of Endocrinology 2017 (2017) 9.

[13] R. Colas, V. Pruneta-Deloche, M. Guichardant, C. Luquain-Costaz, C. Cugnet-Anceau, M. Moret, H. Vidal, P. Moulin, M. Lagarde, and C. Calzada, Increased lipid peroxidation in LDL from type-2 diabetic patients. Lipids 45 (2010) 723-731.

[14] N.E. Braverman, and A.B. Moser, Functions of plasmalogen lipids in health and disease. Biochimica et Biophysica Acta (BBA) - Molecular Basis of Disease 1822 (2012) 1442-1452.

[15] C. Jang, S.F. Oh, S. Wada, G.C. Rowe, L. Liu, M.C. Chan, J. Rhee, A. Hoshino, B. Kim, A. Ibrahim, L.G. Baca, E. Kim, C.C. Ghosh, S.M. Parikh, A. Jiang, Q. Chu, D.E. Forman, S.H. Lecker, S. Krishnaiah, J.D. Rabinowitz, A.M. Weljie, J.A. Baur, D.L. Kasper, and Z. Arany, A branched-chain amino acid metabolite drives vascular fatty acid transport and causes insulin resistance. Nat Med 22 (2016) 421-426.

[16] F.K. Lutchmansingh, J.W. Hsu, F.I. Bennett, A.V. Badaloo, N. McFarlane-Anderson, G.M. GordonStrachan, R.A. Wright-Pascoe, F. Jahoor, and M.S. Boyne, Glutathione metabolism in type 2 diabetes and its relationship with microvascular complications and glycemia. PloS one 13 (2018) e0198626-e0198626.

[17] R.A. Ribeiro, M.L. Bonfleur, T.M. Batista, P.C. Borck, and E.M. Carneiro, Regulation of glucose and lipid metabolism by the pancreatic and extra-pancreatic actions of taurine. Amino Acids 50 (2018) 1511-1524.

[18] X. Liu, J. Gao, J. Chen, Z. Wang, Q. Shi, H. Man, S. Guo, Y. Wang, Z. Li, and W. Wang, Identification of metabolic biomarkers in patients with type 2 diabetic coronary heart diseases based on metabolomic approach. Scientific Reports 6 (2016) 30785.

[19] M. Li, L. Gu, J. Yang, and Q. Lou, Serum uric acid to creatinine ratio correlates with $\beta$-cell function in type 2 diabetes. Diabetes/Metabolism Research and Reviews 34 (2018) e3001.

[20] C.M. Rebholz, A. Surapaneni, A.S. Levey, M.J. Sarnak, L.A. Inker, L.J. Appel, J. Coresh, and M.E. Grams, The Serum Metabolome Identifies Biomarkers of Dietary Acid Load in 2 Studies of Adults with Chronic Kidney Disease. The Journal of Nutrition 149 (2019) 578-585.

[21] S. Luo, J. Coresh, A. Tin, C.M. Rebholz, L.J. Appel, J. Chen, R.S. Vasan, A.H. Anderson, H.I. Feldman, P.L. Kimmel, S.S. Waikar, A. Köttgen, A.M. Evans, A.S. Levey, L.A. Inker, M.J. Sarnak, and M.E. Grams, Serum Metabolomic Alterations Associated with Proteinuria in CKD. Clinical Journal of the American Society of Nephrology 14 (2019) 342-353.

[22] B. Su, and R.O. Ryan, Metabolic biology of 3-methylglutaconic acid-uria: a new perspective. J Inherit Metab Dis 37 (2014) 359-368.

[23] C.B. Newgard, J. An, J.R. Bain, M.J. Muehlbauer, R.D. Stevens, L.F. Lien, A.M. Haqq, S.H. Shah, M. Arlotto, C.A. Slentz, J. Rochon, D. Gallup, O. Ilkayeva, B.R. Wenner, W.S. Yancy, Jr., H. Eisenson, 
G. Musante, R.S. Surwit, D.S. Millington, M.D. Butler, and L.P. Svetkey, A branched-chain amino acid-related metabolic signature that differentiates obese and lean humans and contributes to insulin resistance. Cell Metab 9 (2009) 311-326.

[24] N. Krishnan, M.B. Dickman, and D.F. Becker, Proline modulates the intracellular redox environment and protects mammalian cells against oxidative stress. Free Radic Biol Med 44 (2008) 671-681.

[25] Y.-H. Ham, K.K. Jason Chan, and W. Chan, Thioproline Serves as an Efficient Antioxidant Protecting Human Cells from Oxidative Stress and Improves Cell Viability. Chemical Research in Toxicology 33 (2020) 1815-1821.

[26] S. Sasso, L. Dalmedico, D. Delwing-Dal Magro, A.T. Wyse, and D. Delwing-de Lima, Effect of Nacetylarginine, a metabolite accumulated in hyperargininemia, on parameters of oxidative stress in rats: protective role of vitamins and L-NAME. Cell Biochem Funct 32 (2014) 511-9.

[27] J. Meiser, D. Weindl, and K. Hiller, Complexity of dopamine metabolism. Cell Commun Signal 11 (2013) 34-34.

[28] L.D. Ly, S. Xu, S.-K. Choi, C.-M. Ha, T. Thoudam, S.-K. Cha, A. Wiederkehr, C.B. Wollheim, I.-K. Lee, and K.-S. Park, Oxidative stress and calcium dysregulation by palmitate in type 2 diabetes. Experimental \& molecular medicine 49 (2017) e291-e291.

[29] L.C. Bandet, S. Tan-Chen, O. Bourron, H. Le Stunff, and E. Hajduch, Sphingolipid Metabolism: New Insight into Ceramide-Induced Lipotoxicity in Muscle Cells. International Journal of Molecular Sciences 20 (2019).

[30] S. Xu, S.M. Nam, J.H. Kim, R. Das, S.K. Choi, T.T. Nguyen, X. Quan, S.J. Choi, C.H. Chung, E.Y. Lee, I.K. Lee, A. Wiederkehr, C.B. Wollheim, S.K. Cha, and K.S. Park, Palmitate induces ER calcium depletion and apoptosis in mouse podocytes subsequent to mitochondrial oxidative stress. Cell death \& disease 6 (2015) e1976-e1976. 